\title{
Correction to: Genetic architecture of cardiometabolic risks in people living with HIV
}

\author{
Haoxiang Cheng ${ }^{1 \dagger}$, Anshuman Sewda ${ }^{1,2+}$, Carla Marquez-Luna ${ }^{3}$, Sierra R. White ${ }^{1}$, Bridget M. Whitney ${ }^{4}$, \\ Jessica Williams-Nguyen ${ }^{4}$, Robin M. Nance ${ }^{1,5}$, Won Jun Lee ${ }^{1}$, Mari M. Kitahata, ${ }^{5,}$, Michael S. Saag ${ }^{7}$, Amanda Willig ${ }^{7}$, \\ Joseph J. Eron ${ }^{8}$, W. Christopher Mathews ${ }^{9}$, Peter W. Hunt ${ }^{10}$, Richard D. Moore ${ }^{11,12}$, Allison Webel ${ }^{13}$, \\ Kenneth H. Mayer ${ }^{14}$, Joseph A. Delaney ${ }^{4}$, Paul K. Crane ${ }^{5}$, Heidi M. Crane ${ }^{5,6}$, Ke Hao $^{1+}$ and Inga Peter ${ }^{1 * \dagger}$
}

\section{Correction to: BMC Med 18, 288 (2020) https://doi.org/10.1186/s12916-020-01762-z}

The original article [1] contained an error whereby first author, Haoxiang Cheng's name was displayed incorrectly. This has since been corrected.

\section{Author details}

'Department of Genetics and Genomic Sciences, Icahn School of Medicine at Mount Sinai, 1425 Madison Avenue, New York, NY 10029, United States of America. ${ }^{2}$ Institute of Health Management Research, IIHMR University, Jaipur, Rajasthan, India. ${ }^{3}$ The Charles Bronfman Institute for Personalized Medicine, Icahn School of Medicine at Mount Sinai, New York, NY, United States of America. ${ }^{4}$ Department of Epidemiology, University of Washington School of Public Health, Seattle, WA, United States of America. ${ }^{5}$ Department of Medicine, University of Washington School of Medicine, Seattle, WA, United States of America. ${ }^{6}$ Center for AIDS Research, University of Washington, Seattle, WA, United States of America. ${ }^{7}$ School of Medicine, University of Alabama at Birmingham, Birmingham, AL, United States of America. ${ }^{8}$ Department of Medicine, School of Medicine, University of North Carolina at Chapel Hill, Chapel Hill, NC 27514, United States of America. ${ }^{9}$ Department of Medicine, University of California San Diego, San Diego, CA, United States of America. ${ }^{10}$ Division of Experimental Medicine, University of California San Francisco, San Francisco, CA, United States of America. ${ }^{11}$ Department of Medicine, Johns Hopkins University, Baltimore, MD, United States of America. ${ }^{12}$ Department of Epidemiology, |Johns Hopkins University, Baltimore, MD, United States of America. ${ }^{13}$ Frances Payne Bolton School of Nursing, Case
Western Reserve University, Cleveland, OH, United States of America. ${ }^{14}$ The Fenway Institute at Fenway Health, Boston, MA, United States of America.

Published online: 05 May 2021

\section{Reference}

1. Cheng $\mathrm{H}$, et al. Genetic architecture of cardiometabolic risks in people living with HIV. BMC Med. 2020;18:288. https://doi.org/10.1186/s12916-020-01762-

The original article can be found online at https://doi.org/10.1186/s12916020-01762-z

*Correspondence: inga.peter@mssm.edu

Haoxiang Cheng and Anshuman Sewda are first authors equally contributed to this work.

${ }^{+} \mathrm{Ke}$ Hao and Inga Peter are senior authors equally contributed to this work. 'Department of Genetics and Genomic Sciences, Icahn School of Medicine at Mount Sinai, 1425 Madison Avenue, New York, NY 10029, United States of America

Full list of author information is available at the end of the article

(c) The Author(s). 2021 Open Access This article is licensed under a Creative Commons Attribution 4.0 International License, which permits use, sharing, adaptation, distribution and reproduction in any medium or format, as long as you give appropriate credit to the original author(s) and the source, provide a link to the Creative Commons licence, and indicate if changes were made. The images or other third party material in this article are included in the article's Creative Commons licence, unless indicated otherwise in a credit line to the material. If material is not included in the article's Creative Commons licence and your intended use is not permitted by statutory regulation or exceeds the permitted use, you will need to obtain permission directly from the copyright holder. To view a copy of this licence, visit http://creativecommons.org/licenses/by/4.0/ The Creative Commons Public Domain Dedication waiver (http://creativecommons.org/publicdomain/zero/1.0/) applies to the data made available in this article, unless otherwise stated in a credit line to the data. 